\title{
Mikrobielle Elektrosynthese
}

\section{Bioplastikproduktion mithilfe eines extremophilen kathodischen Biofilms}

\author{
TOBIAS JUNG ${ }^{1}$, MAX HACKBARTH ${ }^{2}$, HARALD HORN ${ }^{2}$, JOHANNES GESCHER ${ }^{1,3}$ \\ 1 INSTITUT FÜR ANGEWANDTE BIOWISSENSCHAFTEN, KARLSRUHER INSTITUT FÜR \\ TECHNOLOGIE (KIT) \\ 2 ENGLER-BUNTE-INSTITUT, TEILINSTITUT WASSERCHEMIE UND WASSERTECHNOLOGIE, \\ KARLSRUHER INSTITUT FÜR TECHNOLOGIE (KIT) \\ 3 INSTITUT FÜR BIOLOGISCHE GRENZFLÄCHEN (IBG-1), KARLSRUHER INSTITUT FÜR \\ TECHNOLOGIE (KIT)
}

As the atmospheric $\mathrm{CO}_{2}$ concentrations are increasing, its usage as biotechnological substrate becomes a focus area of applied scientists. As a rather new technique to energize the process of $\mathrm{CO}_{2}$ fixation, microbial electrosynthesis offers the advantage to establish continuous processes based on a cathodic biofilm that is supplied with electrical energy provided by renewable resources. Here we present the cathodic biofilm growth of Kyrpidia spormannii, a recently isolated thermophilic organism that is naturally capable of producing the biodegradable biopolymer polyhydroxybutyrate (PHB).

DOI: $10.1007 / \mathrm{s} 12268-020-1485-0$

C) Die Autoren 2020

Die anthropogenen Kohlenstoffdioxidemissionen sind heutzutage die größte Gefahr für das Weltklima und gelten als eine der Hauptursachen des Klimawandels. Daher

ist die Erforschung und Weiterentwicklung von Technologien zur stofflichen Nutzung von $\mathrm{CO}_{2}$ für eine zirkuläre, biobasierte Wirtschaft eminent wichtig. Der Einsatz von Mikroorga-
Abb. 1: Schematische Darstellung der mikrobiellen Elektrosynthese (MES) und eines potenziellen Prozesses zur nachhaltigen Produktion höherwertiger Kohlenwasserstoffe wie Polyhydroxybutyrat (PHB). Hier dienen emittiertes Rauchgas zusammen mit nachhaltig generiertem Strom als Substrate.

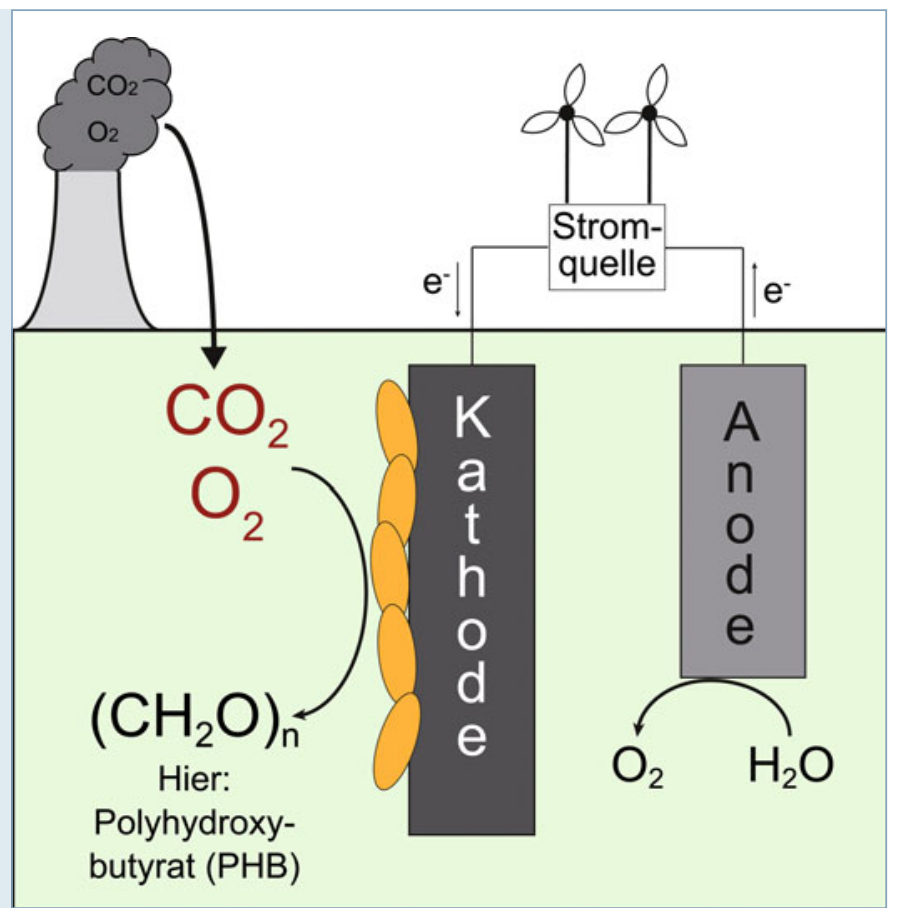

nismen als Biokatalysatoren zur Umwandlung von $\mathrm{CO}_{2}$ in langkettige, energiereiche Verbindungen gilt dabei aufgrund ihrer vielseitigen Einsetzbarkeit als eine der vielversprechendsten Technologien. Neben einer Reihe möglicher Energiequellen wie Licht oder Wasserstoff, ist seit kurzem ebenfalls bekannt, dass bestimmte Mikroorganismen in der Lage sind, Elektronen von einer Kathode als Energiequelle für die Fixierung von $\mathrm{CO}_{2}$ zu nutzen, um Biomasse aufzubauen (Abb. 1). Diese mikrobielle Elektrosynthese (MES) hat daher das Potenzial, eine nachhaltige Technologie zu werden, um zum einen $\mathrm{CO}_{2}$ als günstigen und ubiquitär anfallenden Rohstoff zu nutzen und zum anderen fluktuierend anfallende elektrische Energie aus erneuerbaren Energiequellen zu speichern. Bisher bekannte $\mathrm{CO}_{2}$-assimilierende Biokathoden beruhen oft auf der Verwendung von mikrobiellen Konsortien, wohingegen nur eine geringe Anzahl von Reinkulturen bekannt sind. Diese fallen jedoch primär in die Gruppe der acetogenen und methanogenen Mikroorganismen - eine Organismengruppe, welche nur eine geringe metabolische Variabilität besitzt und strikt anaerobe sowie meist mesophile Organismen beinhaltet. Die meisten industriellen Emissionsquellen für $\mathrm{CO}_{2}$ sind jedoch heiße und Sauerstoff enthaltende Rauchgase, für deren direkte Nutzung sauerstofftolerante und extremophile Organismen benötigt werden. Eine aufwendige Gaskühlung und -aufreinigung wäre für einen biotechnologischen Prozess somit überflüssig und gleichzeitig würde das Risiko einer Kontamination verringert werden.

\section{Kyrpidia spormannii als} Biokatalysator

Eines unserer Ziele war daher die Isolierung eines geeigneten Biokatalysators aus der Anreicherung einer extremophilen Biokathode. Dafür wurden Proben aus heißen Quellen der Insel São Miguel auf den Azoren genommen, um einen Elektrosynthese-Reaktor über mehrere Monate unter thermoacidophilen Bedingungen $\left(60^{\circ} \mathrm{C}\right.$ und $\left.\mathrm{pH} 3,5\right)$ zu kultivieren. Eine Graphit-Kathode (-500 mV vs. Stan- 
dardwasserstoffelektrode, SHE) war dabei die einzige Energie- und $\mathrm{CO}_{2}$ die alleinige Kohlenstoffquelle. Schlussendlich konnte mit Kyrpidia spormannii ein bisher noch nicht beschriebenes, thermoacidophiles und obligat aerob wachsendes Knallgasbakterium isoliert werden [1]. Es war anschließend möglich, auch die elektroautotrophe Lebensweise der Reinkultur in einem bioelektrochemischen Reaktor nachzuweisen. Im Vergleich zu einer abiotischen Kontrolle zeigte K. spormannii einen ca. 5 fach höheren Stromverbrauch, welcher durch vergleichende Transkriptom- und ${ }^{13} \mathrm{C}$-Markierungsversuche auf einen distinkten biologisch-katalysierten Effekt zurückgeführt werden konnte [2]. Das Interesse an der Nutzung dieses Organismus war auch darin begründet, dass es sich bei $K$. spormannii um einen natürlichen Produzenten des Biopolymers Polyhydroxybutyrat (PHB) handelt. Dieses wird von einer Reihe Mikroorganismen als intrazellulärer Kohlenstoff- und Energiespeicherstoff akkumuliert und gilt aufgrund seiner biologischen Abbaubarkeit und Biokompatibilität als vielversprechendes Produkt biotechnologischer Prozesse [3]. Gegenwärtig werden in der industriellen, biotechnologischen PHB-Produktion kostenintensive Substrate wie Glucose und Fructose verwendet [4]. Daher rückte die Nutzung des nachhaltigen und günstigen Rohstoffs $\mathrm{CO}_{2}$ mittels autotropher Mikroorganismen in den Fokus. Die MES könnte Vorteile gegenüber bisher verwendeten wasserstoff- oder licht-basierten Prozessen bieten, da sich hier biofilmbasierte, kontinuierliche Prozesse entwickeln lassen. Dabei muss der Elektronendonor nicht in Lösung gebracht werden und es können große Elektrodenflächen in ein Reaktorvolumen integriert werden. Um nachzuweisen, ob sich $K$. spormannii als PHB-Produzent in einem MES-System eignet, wurde sein kathodisches Biofilmwachstum untersucht.

\section{Kathodisches Biofilmwachstum}

Aus diesem Grund wurden Fließzellen konstruiert, die es erlauben, die akkumulierte Biomasse des Biofilms mittels der optischen Kohärenztomographie (OCT) zu quantifizieren (Abb. 2A, [5]). Durch eine transparente Fließzellenabdeckung kann dies nicht-invasiv und in vivo durchgeführt werden. Dabei kann zum einen der Biofilm morphologisch mithilfe generierter Höhenprofile beschrieben und zum anderen können quantitative Aussagen über akkumulierte Biomasse getroffen werden [6]. Für $K$. spormannii zeigte sich ein maximales mittleres Biovolumen von ca. $65 \mu \mathrm{m}^{3} \mu \mathrm{m}^{-2}$ nach einer 10-tägigen Kultivierung (Abb. 2B). Das akkumulierte Biovolumen war zusätzlich abhängig von der Überströmgeschwindigkeit der Kathode, ein für Biofilme typisches Verhalten [5]. Die Akkumulation erfolgte zu einem Großteil innerhalb der ersten vier Tage und erreichte eine maximale Akkumulationsrate von ca. $14 \mu \mathrm{m}^{3} \mu \mathrm{m}^{-2} \mathrm{~d}^{-1}$. Etwa zu diesem Zeit-

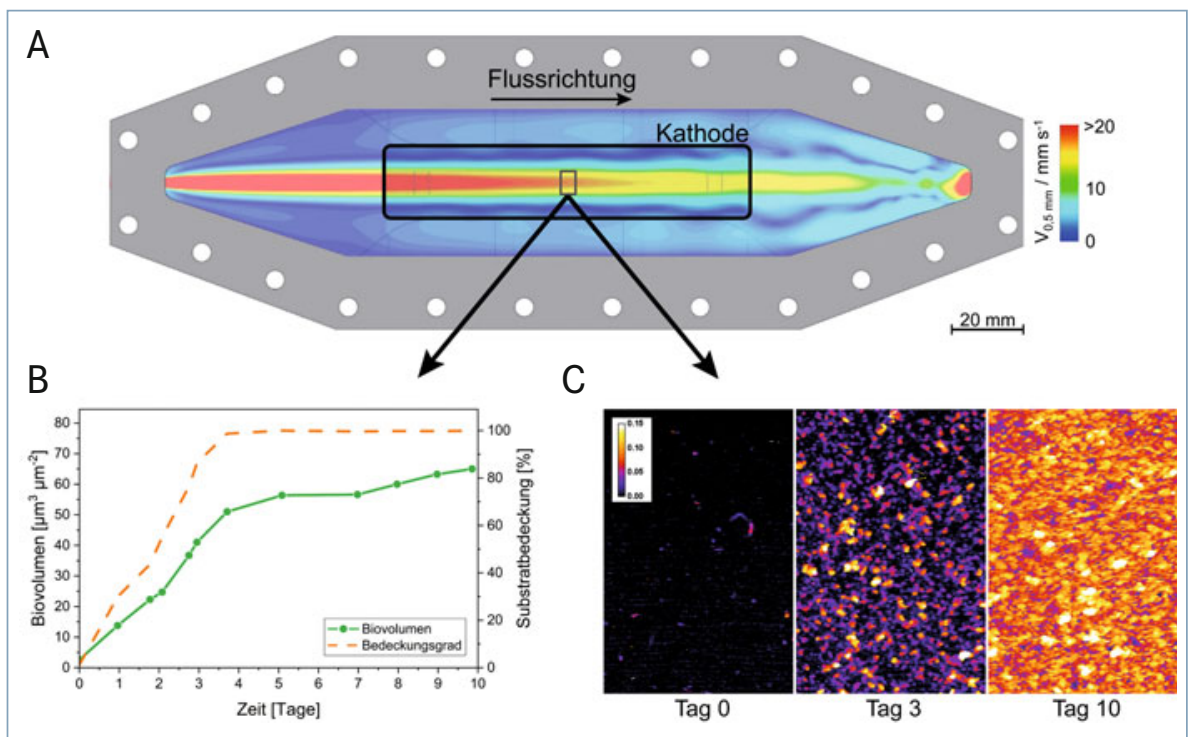

$\Delta$ Abb. 2: Kathodisches Biofilmwachstum von Kyrpidia spormannii. A, OCT-Aufnahmen der Kathodenoberfläche. Die Farbgebung kennzeichnet die simulierte Überströmungsgeschwindigkeit in der Fließzelle. Der Bereich der Kathode ist als schwarzes Rechteck gekennzeichnet. B, Bestimmung von Biofilm-Wachstumsparametern, wie akkumuliertes Biovolumen und Substratbedeckung. C, morphologische Aussagen des Biofilms mithilfe von Höhenprofilen. punkt war die Kathode auch vollständig mit Biofilm bedeckt (Abb. 2B). Dieser Anstieg der Kathodenbedeckung und die relativ gleichmäßige Morphologie des entstehenden Biofilms kann auch in den generierten Höhenprofilen beobachtet werden (Abb. 2C). Das gleichzeitige Sinken der Akkumulationsrate mit der vollständigen Bedeckung der Kathode ist möglicherweise auf eine Elektronentransfer-Limitation im Biofilm zurückzuführen. Wie genau die Elektronenaufnahme von K. spormannii von einer Kathode erfolgt, konnte jedoch noch nicht abschließend geklärt werden. Bisherige Ergebnisse deuten auf eine zumindest teilweise biologisch-katalysierte Wasserstoffentstehung hin, wobei auch die Aufnahme durch sekretierte oder abiotische Redoxmediatoren nicht ausgeschlossen werden kann [2]. Durch fluoreszenzmikroskopische Aufnahmen des Biofilms konnte die intrazelluläre Akkumulation von PHB in Form typischer Granula nachgewiesen werden.

\section{Fazit und Perspektive}

Zusammenfassend kann festgehalten werden, dass sich $K$. spormannii sehr gut als Biokatalysator in einem mikrobiellen Elektrosynthese-Prozess eignet. Das erreichte maximale Biovolumen übersteigt das anderer kathodischer Biofilme von Reinkulturen [7]. Zusätzlich konnte über mikroskopische Analysen die Akkumulation des gewünschten Produkts PHB in den Zellen des Biofilms nachgewiesen werden. Unter welchen Kultivierungsbedingungen die größten Mengen PHB bei elektroautotrophem Wachstum gebildet werden, ist noch Gegenstand aktueller Forschung. Zukünftig sollen die Ergebnisse aus dem Fließzellen-System für eine Hochskalierung genutzt werden. Um dabei ein möglichst großes Verhältnis von Kathodenoberfläche zu Reaktorvolumen bei gleichzeitig optimalen Strömungsbedingungen zu ermöglichen, sollen drehende Kathoden in einen Scheibentauchkörper eingebaut werden. Hierfür ist ein Mechanismus geplant, um die PHB-enthaltende Biomasse kontinuierlich in einem gewissen Maß abzutragen. Dabei soll speziell auf die Realisierbarkeit eines Scale-Ups dieses kontinuierlichen Elektrosynthese-Prozesses zur nachhaltigen Bioplastikproduktion geachtet werden.

\section{Danksagung}

Wir danken dem BMBF (Förderkennziffer 033RC006) für die finanzielle Unterstützung. 


\section{Literatur}

[1] Reiner JE, Jung T, Lapp CJ et al. (2018) Kyrpidia spormannii sp. nov, a thermophilic, hydrogen-oxidizing facultative autotroph, isolated from hydrothermal systems at São Miguel Island, and emended description of the genus Kyrpidia. Int J Syst Evol Microbiol 68:3735-3740

[2] Reiner JE, Geiger K, Hackbarth M et al. (2020) From an extremophilic community to an electroautotrophic production strain: identifying a novel Knallgas bacterium as cathodic biofilm biocatalyst. ISME J 14:1125-1140

[3] Rajan KP, Thomas SP, Gopanna A et al. (2018) Polyhydroxybutyrate (PHB): A standout biopolymer for environmental sustainability. In: Torres Martínez LM, Kharissova OV, Kharisov BI (Hrsg.) Handbook of ecomaterials. Springer International Publishing, Basel, 1-23

[4] Koller M, Salerno A, Reiterer A et al. (2012) Sugarcane as feedstock for biomediated polymer production. In: Goncalves JF, Correia KD (Hrsg.) Sugarcane: production, cultivation and uses. Nova Science Publishers, New York, 105-136

[5] Hackbarth M, Jung T, Reiner JE et al. (2020) Monitoring and quantification of bioelectrochemical Kyrpidia spormannii biofilm development in a novel flow cell setup. Chem Eng J 390:124604

[6] Wagner M, Horn H (2017) Optical coherence tomography in biofilm research: A comprehensive review. Biotechnol Bioeng 114:1386-1402

[7] Salimijazi F, Parra E, Barstow B (2019) Electrical energy storage with engineered biological systems. J Biol Eng 13:38
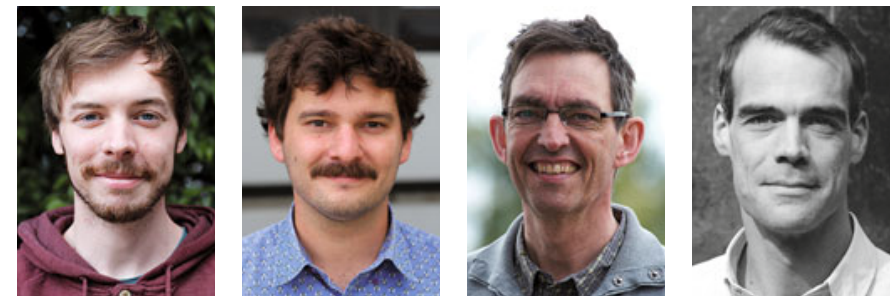

Tobias Jung, Max Hackbarth, Harald Horn und Johannes Gescher (v. I. n. r.)

Funding note: Open Access funding enabled and organized by Projekt DEAL. Open Access: Dieser Artikel wird unter der Creative Commons Namensnennung 4.0 International Lizenzveröffentlicht, welche die Nutzung, Vervielfältigung, Bearbeitung, Verbreitung und Wiedergabe in jeglichem Medium und Forma erlaubt, sofern Sie den/die ursprünglichen Autor(en) und die Quelle ordnungsgemaß nennen, einen Link zur Creative Commons Lizenz beifügen und angeben, ob Anderungen vorgenommen wurden. Die in diesem Artikel genannten Creative Commons Lizenz, sofern sich aus der Abbildungser genannten Creative Commons Lizenz, soferm sich aus der Abbildungsleg genannten Creative Commons Lizenz steht und die betreffende Handlung nich nach gesetzlichen Vorschriften erlaubt ist, ist für die oben aufgeführten Weiterverwendungen des Materials die Einwilligung des jeweiligen Rechteinhabers einzuholen. Weitere Details zur Lizenz entnehmen Sie bitte der
Lizenzinformation auf http://creativecommons.org/licenses/by/4.0/deed.de.
Korrespondenzadresse: Prof. Dr. Johannes Gescher Institut für Angewandte Biowissenschaften (IAB) Karlsruher Institut für Technologie (KIT)

Fritz-Haber-Weg 2

D-76131 Karlsruhe

johannes.gescher@kit.edu www.gescher-lab.de

\section{Hier steht} eine Anzeige. 